\title{
Localization of leucine aminopeptidase isoenzymes
}

\author{
B. W. MEADE AND S. B. ROSALKI \\ From the Group Pathological Laboratory, Kingston upon Thames, Surrey
}

SYNOPSIS A simple, sensitive staining procedure for the localization of leucine aminopeptidase isoenzymes after electrophoretic separation on cellulose acetate membranes is described. Separation of leucine aminopeptidase isoenzymes, using cellulose acetate membranes, does not appear to be a rewarding diagnostic procedure in the investigation of hepatobiliary or pancreatic disorders.

The fractionation of enzymes into isoenzymes by electrophoresis provides a means whereby the diagnostic specificity of many serum enzyme determinations may be enhanced. The normal isoenzyme activity of serum may alter in disease, and the changed pattern may characterize the source in the tissues of this altered activity.

Enzyme staining techniques have placed the demonstration of isoenzymes within the scope of the routine laboratory, and have been applied to the demonstration of lactate dehydrogenase (Latner and Skillen, 1961; Van der Helm, Zondag, Hartog, and Van der Kooi, 1962) and alkaline phosphatase (Hodson, Latner, and Raine, 1962) isoenzymes. Smith and Rutenburg (1963) have separated the isoenzyme fractions of leucine aminopeptidase by electrophoresis on cellulose acetate membranes. They demonstrated these by cutting the membranes into segments and subsequently eluting and assaying the activity of each fraction. The present communication describes a simple and sensitive staining method of localizing the leucine aminopeptidase isoenzymes, and also its application to the study of certain aspects of hepatobiliary disease.

\section{PRINCIPLE}

Leucine aminopeptidase is separated into its component isoenzymes by electrophoresis on cellulose acetate membranes. Using a layering technique (Barnett, 1962), the isoenzymes are incubated with L-leucyl- $\beta$-naphthylamide and the $\beta$-naphthylamine subsequently liberated is coupled with an azo-dye, 4 amino - 3:1' dimethyl azobenzene (Fast Garnet G.B.C.), to give pink staining bands in the areas of activity.

Received for publication 7 May 1963.

\section{MATERIAL AND METHODS}

Sera and tissue homogenates ( 5 to $10 \mu l$.) are separated on $12 \times 5 \mathrm{~cm}$. Oxoid cellulose acetate membranes in 0.07 M barbitone buffer, $p \mathrm{H} 8.6$, for 75 minutes, using a constant current of 0.5 milliamps per strip of $1 \mathrm{~cm}$. wide. Tissue homogenates are prepared in deionized water and diluted to yield leucine aminopeptidase activities (Goldbarg, Pineda, and Rutenburg, 1959) between 100 and 500 Klett units (24 to 120 international units per litre of serum at $37^{\circ} \mathrm{C}$.).

After separation the cellulose acetate is divided longitudinally and one half strip is immediately layered onto the surface of a $12 \times 5 \mathrm{~cm}$. strip of Whatman no. 54 filter paper, well moistened with buffer substrate solution $(0.75 \mathrm{ml}$. of $0.2 \mathrm{M}$ phosphate buffer, $p \mathrm{H} 5.7$ and $0.75 \mathrm{ml}$. of a solution of L-leucyl- $\beta$-naphthylamide $0.8 \mathrm{mg}$. per $\mathrm{ml}$. deionized water) and previously placed on a clean glass plate inside a moist chamber. The moist chamber and its contents are incubated at $37^{\circ} \mathrm{C}$. for one hour, during which time the remaining cellulose acetate half strip is dried and stained for protein with Ponceau $S$.

At the end of incubation the cellulose acetate from the moist chamber is surface dried using a hot-air blower and allowed to soak up a solution of Fast Garnet G.B.C. (1 mg. per ml. deionized water). The half strip is placed on a clean glass plate inside a moist chamber and reincubated for 15 minutes. Leucine aminopeptidase isoenzymes show up as pink-staining bands.

The technique outlined above was found to be sensitive to as little as 10 to $20 \mathrm{Klett}$ units of leucine aminopeptidase activity, representing the detection of some 1 to $2 \mu \mathrm{g}$. of liberated $\beta$-naphthylamine. Sensitivity was slightly superior to that obtained by elution of isoenzymes from the cellulose acetate by the technique of Smith and Rutenburg (1963). No increased sensitivity was obtained by the inclusion of cyanide $(0.003 \mathrm{M})$, manganese $(0.01$ $\mathrm{M})$, or magnesium $(0.01 \mathrm{M})$ ions in the buffer-substrate mixture.

The low $p H(5.7)$ of the buffer-substrate mixture was found to give optimal sensitivity, presumably by counteracting the higher $p \mathrm{H}(8 \cdot 6)$ of the barbitone buffer, used to 
obtain good protein separation. Impregnating filter paper with this buffer-substrate solution yielded greater sensitivity than the impregnation of cellulose acetate (as used in lactate dehydrogenase layering techniques). Inclusion of the dye (Fast Garnet G.B.C.), used for the detection of the liberated $\beta$-naphthylamine in the buffer-substrate solution, resulted in marked inhibition of isoenzyme leucine aminopeptidase activity.

To ensure that no staining artefacts were being encountered the whole procedure was repeated for the sera and extracts substituting deionized water for the substrate. No staining of these controls was obtained.
RESULTS

The results of leucine aminopeptidase isoenzyme $\vec{F}$ staining after separation of normal and pathological $\stackrel{\text { ? }}{?}$ sera and of tissue homogenates are detailed in the Table and illustrated in the Figure.

Normal sera, liver, and bile show a single staining

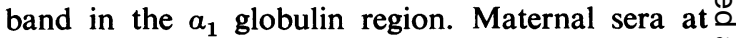
term and placenta show a single band in the $\alpha_{2} \infty$ globulin. Kidney tissue presents a broad band in the $\vec{\circ}$ $\beta-\alpha_{2}$ region and pancreas a single band in the $\beta$.

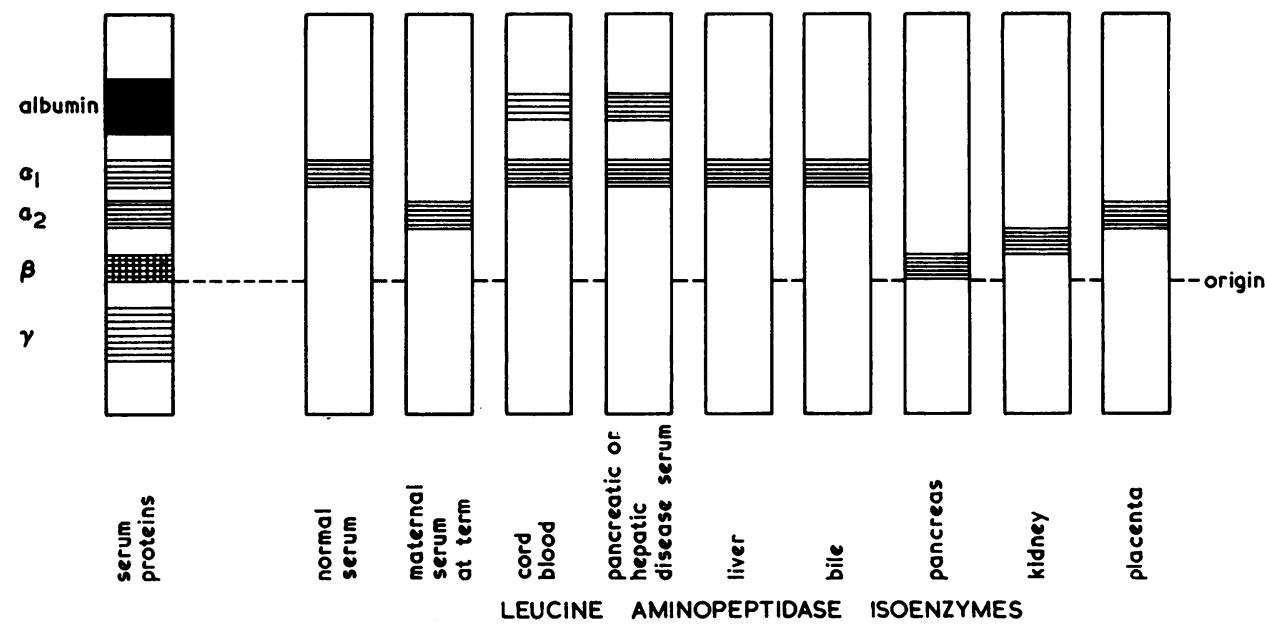

TABLE

RESULTS OF LEUCINE AMINOPEPTIDASE STAINING IN NORMAL AND PATHOLOGICAL SERA

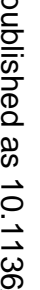

Number

Examined
Activity in Klett Units
Isoenzyme Bands

\begin{tabular}{lllll}
\hline Albumin & $\alpha_{1}$ & $\alpha_{2}$ & $\beta$ & $\gamma$
\end{tabular}

\section{.}

Normal sera

Liver

Bile (diluted)

Pancreas

Kidney

Placenta

Cord blood

Pregnancy serum at term

Serum from carcinoma pancreas Raised Normal

Serum from acute pancreatitis Raised

Normal

Serum from hepatic metastases

Raised

Normal

Serum from hepatic cirrhosis Raised

Serum from acute hepatitis Raised

Normal

Serum from obstructive jaundice Raised

'No. observed.

\begin{tabular}{|c|c|c|c|c|c|}
\hline $96-128$ & - & $+(10)$ & - & - & - \\
\hline $130-280$ & - & $+(3)$ & - & - & - \\
\hline $270-352$ & - & $+(3)$ & - & - & - \\
\hline $235-285$ & - & - & - & - & - \\
\hline 204-293 & - & - & - & $+(3)$ & - \\
\hline $114-500$ & - & - & $+(3)$ & - & - \\
\hline 114-205 & $+(3)^{1}$ & $+(3)$ & - & - & 一 \\
\hline $535-910$ & - & - & $+(5)$ & - & - \\
\hline 370 & $+(1)$ & $+(1)$ & - & - & - \\
\hline 156 & - & $+(1)$ & - & - & - \\
\hline 205 & $+(1)$ & $+(1)$ & - & - & - \\
\hline 155 & $+(1)$ & $+(1)$ & - & - & - \\
\hline $201-500$ & $+(2)$ & $+(4)$ & - & - & - \\
\hline $102-138$ & $+(1)$ & $+(4)$ & 一 & - & - \\
\hline $235-250$ & $+(2)$ & $+(2)$ & - & - & - \\
\hline $205-330$ & $+(1)$ & $+(3)$ & - & - & - \\
\hline 142 & - & $+(1)$ & - & - & - \\
\hline $290-880$ & $+(6)$ & $+(8)$ & - & - & - \\
\hline
\end{tabular}

$290-880+(6)+(8)$ 
globulin. Umbilical cord bloods and sera from a variety of hepatobiliary and pancreatic disorders have two staining bands, the most prominent in the $\alpha_{2}$ globulin, and a second, fainter band in the albumin region. In sera from obstructive jaundice this second band may stain more intensely than the $a_{1}$ band.

\section{DISCUSSION}

The results obtained by the staining technique above are similar to those obtained by elution from cellulose acetate as described by Smith and Rutenburg (1963).

It was not found possible to demonstrate any leucine aminopeptidase isoenzyme activity in the $\gamma$ globulin region in sera or tissue extracts, and no pathological serum examined either by staining or elution showed any activity in the $\beta$ globulin region. Increased isoenzyme activity in the albumin region was observed as a non-specific finding of little diagnostic value in a variety of hepatobiliary and pancreatic disorders.

Smith and Rutenburg (1963) have described leucine aminopeptidase isoenzyme activity in the $\beta$ globulin region in serum from a case of hepatic metastases, and increased isoenzyme activity in the $\beta$ region and adjacent to the origin have been observed in sera from hepatobiliary disorders and pancreatic disease separated on paper (Smith, Pineda, and Rutenburg, 1962) and on starch gel (Kowlessar, Haeffner, and Sleisenger, 1960). Our findings with cellulose acetate indicate that the incidence of increased leucine aminopeptidase isoenzyme activity in the $\beta$ (or $\gamma$ ) region must be too low to be of value in the diagnosis of hepatic metastases or pancreatic disease. The demonstration of leucine aminopeptidase isoenzymes after separation on cellulose acetate would not, therefore, appear to be a rewarding diagnostic procedure for these conditions.

\section{REFERENCES}

Barnett, H. (1962). Lancet, 2, 199.

Goldbarg, J. A., Pineda, E. P., and Rutenburg, A. M. (1959). Amer. J. clin. Path., 32, 571.

Hodson, A. W., Latner, A. L., and Raine, L. (1962). Clin. chim. Acta, 7, 255.

Kowlessar, O. D., Haeffner, L. J., and Sleisenger, M .H. (1960). J. clin. Invest., 39, 671.

Latner, A. L., and Skillen, A. W. (1961), Lancet, 2, 1286.

Smith, E. E., Pineda, E. P., and Rutenburg, A. M. (1962). Proc. Soc. exp. Biol. (N.Y.), 110, 683.

- and Rutenburg, A. M. (1963). Nature (Lond.), 197, 800.

Van der Helm, H. J., Zondag, H. A., Hartog, H. A. Ph., and Van der Kooi, M. W. (1962). Clin. chim. Acta, 7, 540. 